\title{
Design and Implementation of Service Provider for Integration of Heterogeneous Sensor Networks
}

\author{
Chen $\mathrm{Nan}^{1}$, Faiza Tila ${ }^{2}$ and Do Hyeun $\mathrm{Kim}^{2 *}$ \\ ${ }^{1}$ KETI, 25, Saenari-ro, Bundang-gu, Seongnam-si, Gyeonggi-do, 463-816 Korea \\ ${ }^{2}$ Dept. of Computer Engineering, Jeju National University, 690-756 Jeju-si, Korea \\ 1xuehu001@gmail.com, ${ }^{2}$ faizakhan797@gmail.com, \\ ${ }^{2}$ kimdh@jejunu.ac.kr
}

\begin{abstract}
Recently, there is a growing IoT(Internet of Things) service using sensors for environmental monitoring and actuators for reacting to environmental changes. Sensor networks have provided us with facilities to collect information and track certain phenomena in the physical environment based on the region of interest. Sensor networks consist of a collection of sensor nodes and a sink node for many applications in different areas, including environmental surveillance, intelligent building, health monitoring, intelligent transportations, etc. In this paper, we present a sensor service provider for integration of heterogeneous large-scale sensor networks. This paper focuses on the design and implementation of the open sensor service provider for the integrated multiple sensor middleware in heterogeneous sensor networks. This provider has various heterogeneous sensor middleware, and each sensor middleware has a sensor networks. We design the sensor service provider using sequence diagram and state diagram, and implement it based on .Net framework. The sensor service provider has the service interface, the sensor content service manager, the sensor service manager, the sensing service manager, and the service controller, etc. The provider supports comprehensive to carry out the context acquisition and provisioning functionality.
\end{abstract}

Keywords: Sensor networks, sensor service provider, sensor network middleware

\section{Introduction}

Recently, a lot of sensors are deployed to monitor the context in heterogeneous environments. A sensor is an engineering point of view as a device that collect the phenomenon signal. A sensor is defined from an engineering point of view as a device that converts a physical, chemical, or biological parameter into an electrical signal [1]. Common examples include sensors for measuring temperature (i.e., a thermometer), wind speed (an anemometer) conductivity, or solar radiation. While a sensor is the most basic unit, a sensor system is an aggregation of sensors, attached to a single platform [2]. Examples are a weather station with attached sensors, or a combination of heart frequency and blood pressure sensors carried by a human or animal. A sensor or a sensor system may be abstracted as a sensor resource. A sensor network consists of a number of spatially distributed and communicating sensor resources [3, 4].

Sensor networks consist of small nodes communicated with each other, and perform distributed computations in order to monitor phenomena in environment. Sensor networks compose to various configuration according to number of nodes, limited resources, and unreliable communication links.

Sensor networks have provided us with facilities to collect information and track certain phenomena in the physical environment based on the region of interest. Sensor networks consist of a collection of sensor nodes and a sink node for many applications in 
different areas, including environmental surveillance, intelligent building, health monitoring, intelligent transportations.

The sensor middleware is to enable clients to view context data on Internet. Sensor network middleware is providing the sensing-based pervasive computing applications using a sink node and the sensor nodes.

The term sensor web was first used by Kevin Delin of NASA in 1997, to describe a novel wireless sensor network architecture where the individual pieces could act and coordinate as a whole. Delin's definition of a sensor web was an autonomous, stand-alone, sensing entity - capable of interpreting and reacting to the data measured - that does not necessarily require the presence of the World Wide Web to function [5]. For the availability of intelligent services, technologies such as CPS (Cyber Physical System), IoT (Internet of things), and WoT (Web of things) and Sensor Web have been studied through connecting physical and virtual space. Advance research is being carried out by OGC (Open Geospatial Consortium) related to sensor web. It reports real time context data using information collected from various sensors installed in indoor or outdoor environment. Real time context data is managed on the web using the concept of SOA (Service Oriented Architecture) [6].

In [7] and [8] the sensor service provider is used in a cloud context it states that the main responsibility of a sensor service provider is to detect available sensors, communicate with the sensor owners and obtain permission to publish the sensors in the cloud. Sensor service provider are separate business entities. When a sensor owner registers a specific sensor, sensor service provider collects information about the sensor availability, owner preferences and restriction, expected return and so on. All this information needs to be published in the cloud. Once the registration is done, Sensor service provider waits until a sensor consumer makes a request. When sensor service provider receives such a request, it forwards all the details including the offer to the corresponding sensor owner(s) to accept or reject. If the sensor owner accepts the offer, the corresponding sensor data consumer will be able to acquire data from that sensor through the Sensor service provider during the period mentioned in the agreement.

In this paper, we present a sensor service provider based on integration of multiple sensor middleware in heterogeneous sensor networks. This service provider is connected to the higher application clients through a service interface. Also, we design the sensor service provider for integration of multiple sensor middleware using sequence diagram and state diagram. Also we implement the service provider to connect between the application and the sensor middleware using .Net framework. Accordingly, the service provider interconnects between application and heterogeneous sensor networks efficiently. The service provider can retrieve comprehensive the context data from the sensor middleware in heterogeneous sensor networks.

The rest of the paper is as organized follows. A service provider design for sensor networks are introduced in Section II. Section III illustrates a service provider implementation of sensor networks, and Section IV concludes.

\section{A Service Provider Design for Sensor Networks}

A service provider of sensor networks is a business providing computer-based services to customers on sensor network. The service provider addresses an open platform to develop various applications based on sensor networks.

Figure 1 show an integration architecture of sensor networks included the sensor network, sensor middleware, service provider, and applications. Each sensor network has its own middleware, service provider, app server and client attached to it. Sensor networks are powerful systems that consists of sensor nodes, these nodes works together to accumulate information about environmental characteristics i.e. temperature, motion, 
light etc. Each sensor network handles communication and management of data. It provides functions for getting sensor data, turning sensor on and off .

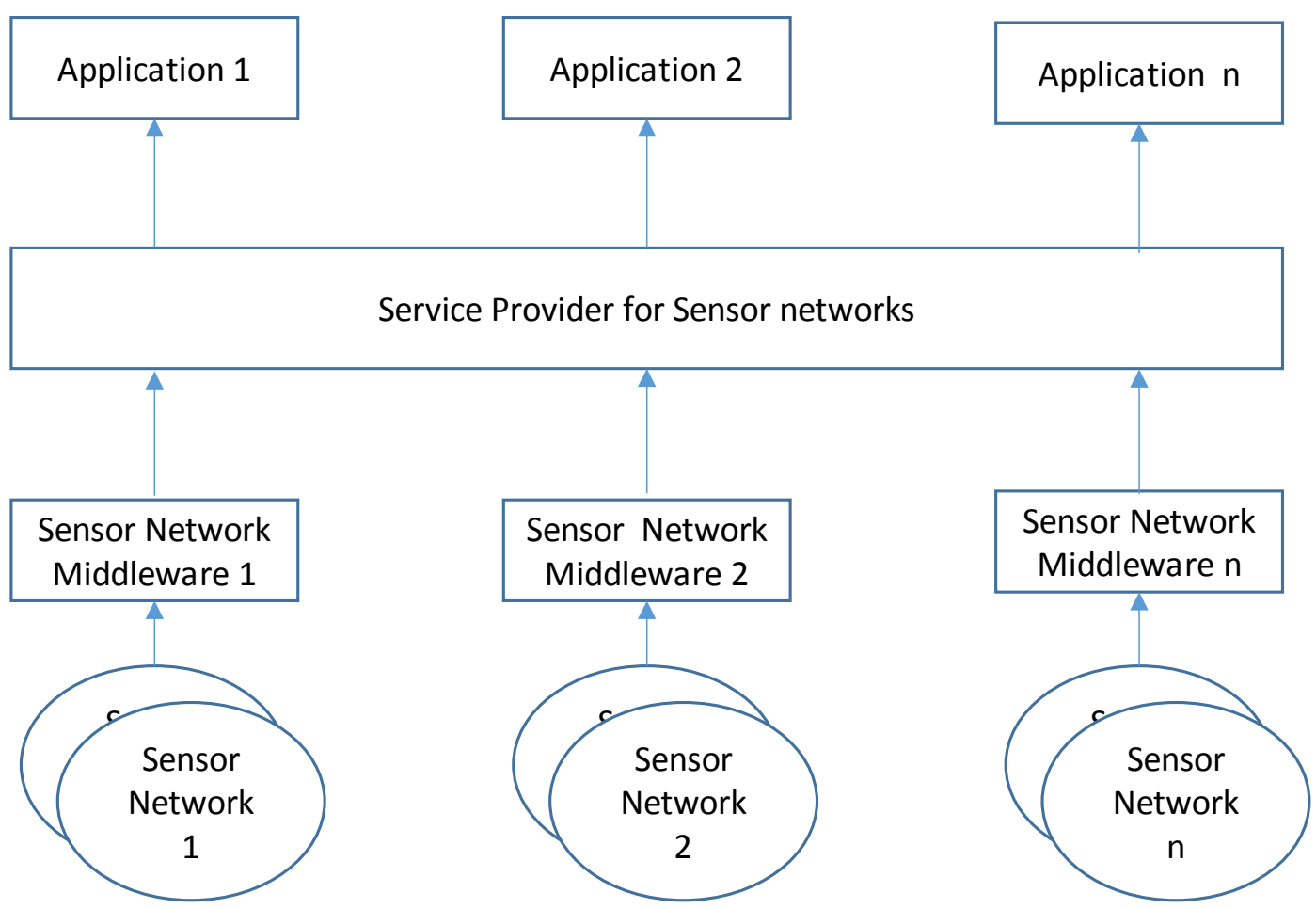

\section{Figure 1. Architecture Based on Sensor Service Provider for Integration of Sensor Networks}

A sensor network middleware collects real-time context data directly from sensors and sends it to the sensor service provider to be stored in the database. It also maintains its own database to store information related to sensors and the data acquired from various sensors.

A service provider for sensor networks offers three major services. First, it also maintains its own database to store information related to sensors and the data acquired from various sensors. Next, the sensing service provided by the provider delivers stored data from the database to other system components. The content service is used for management of sensors and middleware. An application may then request the service provider to get context data for selected sensors and may view it.

Figure 2 illustrates the sensor service provider configuration included modules and sub modules of the service provider for integrated sensor networks. Service interface provides access other system to transfer sensing data. The main interfaces consist of sensing service interface, sensor web content service interface, and sensor provider service interface. The main modules are sensor content service manager, sensor service manager and sensing service manager, and service controller, etc. The sensor content service manager is used for middleware configuration management and sensor information management. The middleware configuration management involves the activities of saving and managing middleware ID, IP addresses and service access privilege information. The sensor information management involves the creation and management of sensor information such as node name, ID and sensing type. 


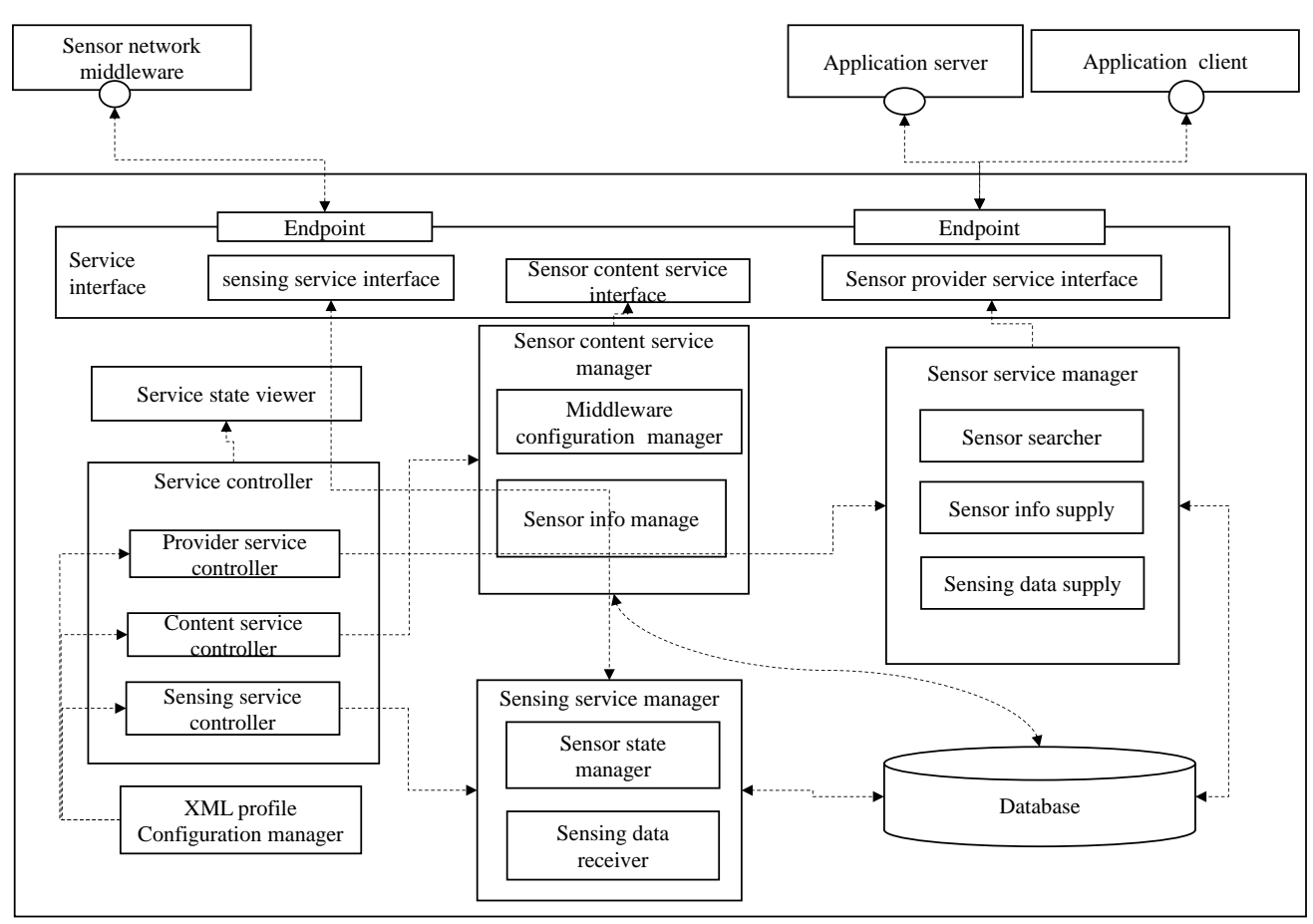

Figure 2. Proposed Service Provider Configuration for Sensor Network

The sensor service manager is utilized for sensor searching, sensor information supply and sensing data supply. The sensor searcher returns sensor ID list for a specific search keyword. The sensor information supply provides related information such as sensor node name, sensing type for a sensor ID. The sensing data supply for a sensor ID, provides the corresponding real time sensing data.

The sensing service manager is used for sensor state manager and sensing data receiver. The sensor state manager involves keeping record of the sensing state of sensors. The sensing data receiver provides the facility of receiving sensing data collected from sensor middleware and saving it. The service controller involves the launching and closing of the provider service controller, sensing service controller, and content service controller. The service state viewer manages a service's state of execution. XML configuration manager provides WCF service state. Database saves sensor related information such as name, ID and sensing data.

Figure 3 shows the interaction of service provider with other system such as middleware, and sensor networks. The sensor service provider has three services namely: sensor provider service, content service and sensing service. The sensor network middleware uses the sensing service to send sensing data to provider. The sensor service provider then uses this data to update its database. The service provider is used by the application to access sensor service provider for sensing data and other sensor related information.

Figure 4 shows state chart for the sensor service provider. It shows the starting sequence of the module and shows how the services are controlled using the controls on the main form of the sensor service provider. First it gets a count of the sensors available. In the next state it start the provider supports namely sensing service which the middleware uses to send sensing data to the provider, content service which the sensor support toolbox uses for management purposes. The service provider provides the application to access for sensing data and other related information. Next we have a decision to take of whether to restart the services or not to goes back to the first state, if yes is taken and closes it. 


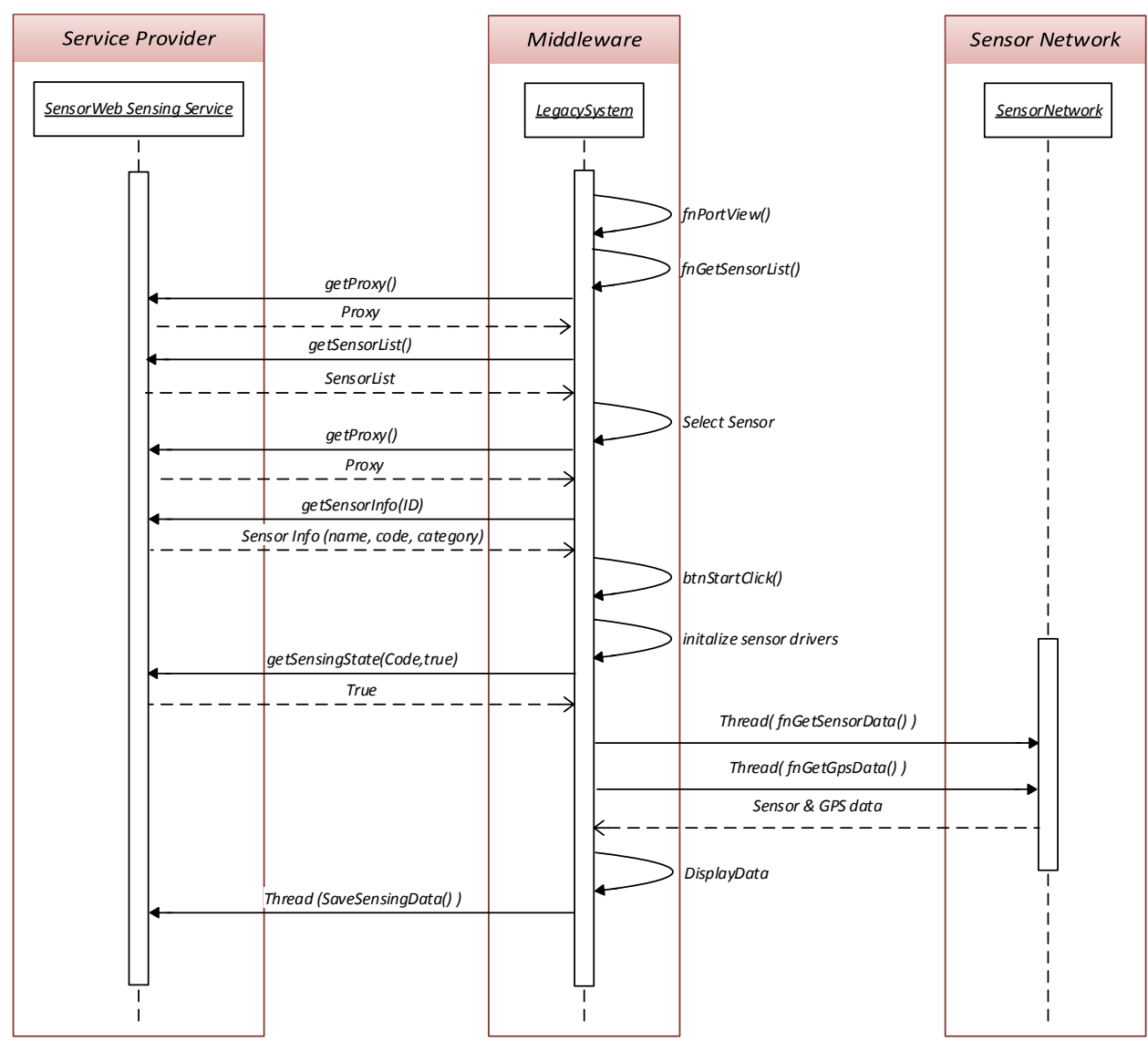

Figure 3. Sequence Diagram of the Service Provider for Sensor Networks

\section{A Service Provider Implementation for Sensor Networks}

The service provider implementation environment is described in table 1 for sensor networks. We use Microsoft Windows 7(X64) for the operating system. Developing is performed in visual studio .net framework version 3.5 or 4. We use Microsoft SQL Server 2008 R2 for database management. Hardware computer has Intel core i 3 with 3.30 Ghz for CPU, 4 GB ram and NVIDIA Geforce GT 440 graphics card.

Figure 5 illustrates implementation result for the service provider of sensor networks. In figure 5, the result shows how the service provider collects the control data and provides it to the other system. And, we have buttons for starting and stopping the provider services. Initially the stop buttons are disabled, they get enabled once the services are started. 


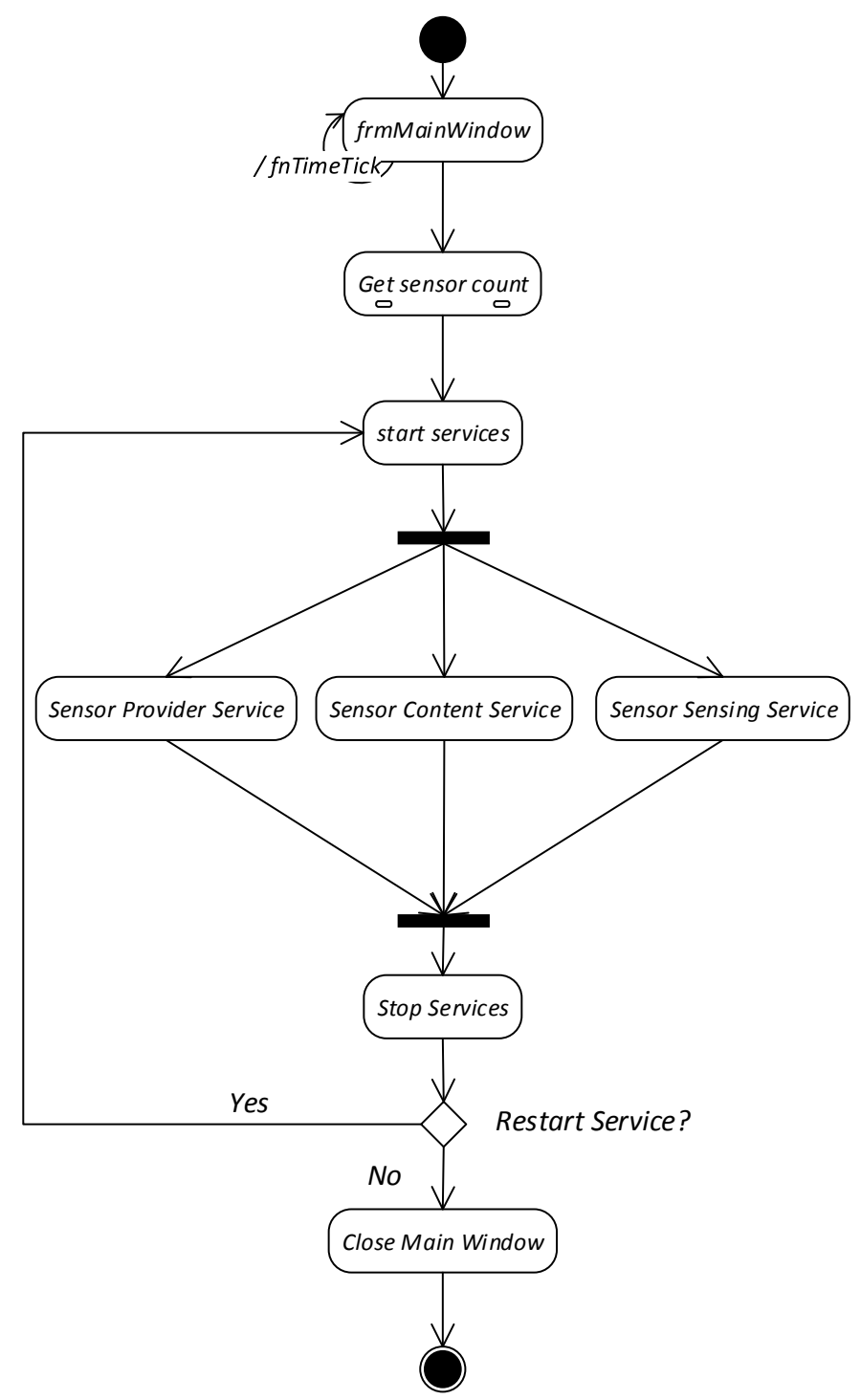

Figure 4. State Diagram for the Service Provider for Sensor Networks

\section{Table 1. Implementation Environment of the Middleware for Sensor} Networks

\begin{tabular}{|l|l|}
\hline & \\
\hline Operating System & Microsoft Windows 7(X64) \\
\hline Development Environment & .Net Framework 3.5, 4.0 \\
\hline Development Tool & Visual Studio .Net 2010 \\
\hline Programming Language & C\#, XMAL, XML \\
\hline DBMS & Microsoft SQL Server 2008 R2 \\
\hline & CPU: Intel® Core ${ }^{\text {TM }}$ i3-2125 @ 3.30GHz \\
Hardware & RAM: 4GB \\
& Graphics: NVIDIA GeForce GT 440 \\
\hline
\end{tabular}

The content service updates the sensor information based on the sensor name it updates the sensor id, sensor explain sensor code and the type code. It shows the sensor information such as sensor code, sensor name, sensor id, sensor explain and type code based on its id, adds new sensor's information and middleware information to the database, deletes sensor information from database table, gets sensor type information, 
gets a list of the sensors and sensing categories of all the sensors, shows a list of all the middle wares information such as middleware code, middleware id, middleware right and config time, adds new middleware to the database. It deletes middleware from table, and updates middleware information table in the database based on the middleware code.

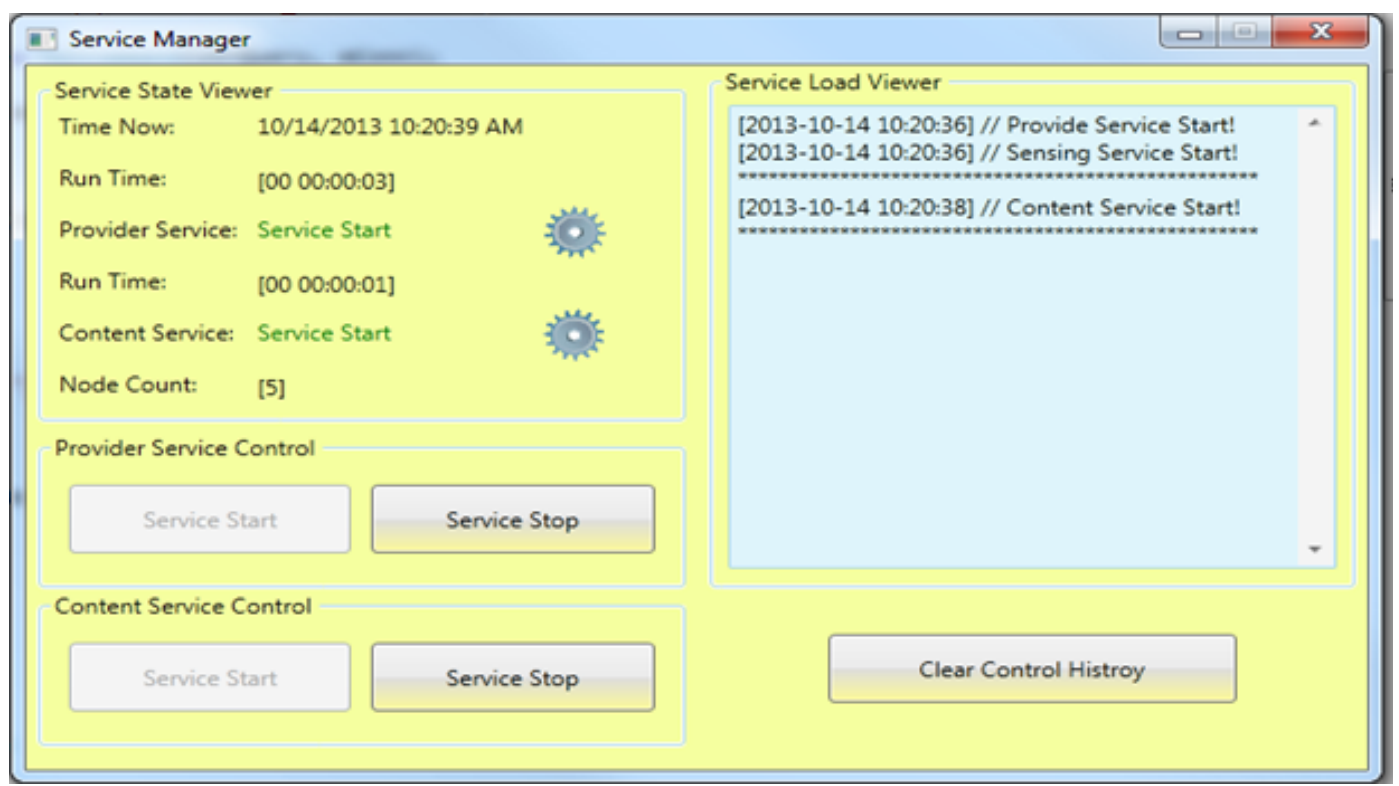

Figure 7. Implementation Result of the Service Provider For Sensor Networks

\section{Conclusion}

In this paper, we present a sensor service provider for integrated multiple sensor network middleware in sensor networks. Also, we design this sensor service provider to connect between application and sensor networks. Also we implement the sensor service provider included the service interface, the sensor content service manager, the sensor service manager and the sensing service manager, and the service controller. The sensor service provider provides comprehensive to transfer sensing data between application and sensor networks.

\section{Acknowledgments}

This work was partly supported by Institute for Information \& communications Technology Promotion(IITP) grant funded by the Korea government(MSIP) (No.10043907, Development of high performance IoT device and Open Platform with Intelligent Software) and This research was supported by the MSIP(Ministry of Science, ICT and Future Planning), Korea, under the ITRC(Information Technology Research Center) support program (IITP-2015-H8501-15-1017) supervised by the IITP(Institute for Information \& communications Technology Promotion) Corresponding author; DoHyeun Kim (e-mail: kimdh@jejunu.ac.kr).

\section{References}

[1] B. Dong, "Design and Implementation of Middleware for Wireless Sensor Networks." Applied Mechanics and Materials, vol. 530, (2014).

[2] C. Stasch, K. Janowicz, A. Bröring, I. Reis and W. Kuhn, "A Stimulus-Centric Algebraic Approach to Sensors and Observations", In Proceedings of 3rd International Conference on Geosensor Networks, (2009). 
[3] T.Z. Van, I, Simonis and G. McFerren,'The Sensor Web: Systems of Sensor Systems”, Int. J. Digital Earth (2009).

[4] A. Bröring, J. Echterhoff, S. Jirka, I. Simonis, T. Everding, C. Stasch, S. Liang and R. Lemmens, "New generation sensor web enablement", vol. 11, no. 3, (2011).

[5] Wikipedia, Sensor Web, retrieved from http://en.wikipedia.org/wiki/Sensor_web.

[6] K.A. Delin, and S.P. Jackson, "Sensor web: a new instrument concept", Symposium on Integrated Optics. International Society for Optics and Photonics, (2001).

[7] Xively, "Xively-ublic cloud for the Internet of Things", rerieved from https://xively.com

[8] Open IoT Consortium, "Open source solution for the internet of things into the cloud", retrieved from http://www.openiot.eu

[9] F.L. Lewis, "Wireless sensor networks." Smart environments: technologies, protocols, and applications (2004).

\section{Authors}

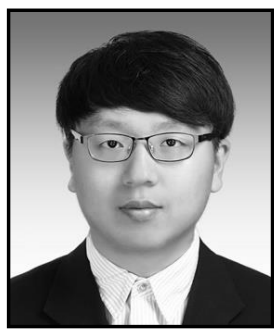

Nan Chen, He received the Master degree in mobile computing from the Jeju National University, Jeju-si, Republic of Korea. Currently, he is a Researcher at Embedded Software Combination Research Center of the KETI(Korea Electronics Technology Institute). His research interests have IoT open platform and IoT Interworking.

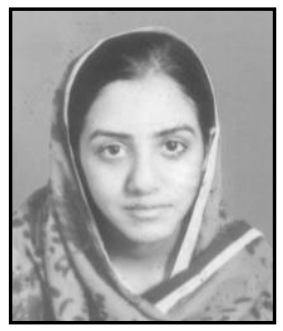

Faiza Khan, She received the B.Sc. degree in Computer Software Engineering from University of Engineering and Technology Pakistan in 2012. She joined the Mobile Computing lab Jeju National University as a M.S. student in 2014. Her area of interest is Semantic Web Technologies and Internet of things.

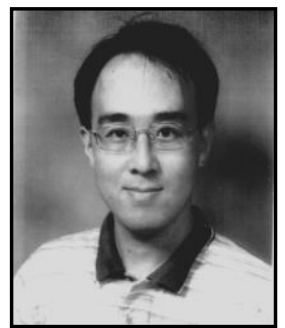

Do-Hyeun Kim, He received the B.S., M.S. and P.D degrees in Electronics Engineering from Kyungpook National University, Taegu, Korea, in 1988 and 1990, 2000 respectively. He joined the Agency of Defense Development (ADD), Korea, in 1990. Since 2004, he is currently a professor at the Department of Computer Engineering at Jeju National University, Korea. His research interests include sensor web, and context prediction. 